\title{
MÉTHODE DIRECTE D'APPRÉCIATION DU TASSÉ DES TOISONS
}

\author{
PAR
}

\section{P. CHARLET}

Maìtre de Conférences de Zootechnie à l'Institut National Agronomique

La densité ou tassé d'une toison est mesurée par le nombre de fibres par unité de surface de la peau : l'unité choisie est, en général, le centimètre carré.

La connaissance de cette valeur offre un grand intérêt pour de nombreuses études. La première notion qu'elle donne est celle du nombre de follicules pileux en fonctionnement. D'après les travaux anglais (notamment ceux de Miss GalPIN), cette valeur varie selon les races et les individus et, pour un même animal, avec l'âge, les régions du corps et la saison. On constate empiriquement que la densité varie avec les races, en raison inverse de la finesse moyenne. Au sein d'une même race, cette relation semble moins constante chez les différents individus.

Cette même étude de densité peut permettre, dans les toisons jarreuses, de connaître la proportion des fibres normales et des poils de jarre. Cette proportion peut varier, pour un même animal, avec l'âge et les conditions de milieu (climat, nourriture, etc.).

C'est encore la mesure (approchée d'ailleurs) de la densité ainsi définie qui a permis de montrer, que, compte tenu des facteurs ci-dessus (âge, conditions de milieu, etc.), la densité de la toison est un facteur héréditaire.

Nous voyons ainsi le grand intérêt que présente la détermination aussi précise que possible de cet important facteur.

De nombreuses méthodes ont été proposées pour mesurer le tassé, que l'on peut diviser en deux groupes : celui des méthodes directes et celui des méthodes indirectes.

\section{MÉTHODES INDIRECTES}

La plus classique parmi ces méthodes, consiste à évaluer, d'une part, la surface couverte par la toison, et, d'autre part, le nombre total de fibres revêtant cette surface. 
Nous pouvons, d'après le poids vif d'un animal, obtenir de façon approximative la surface corporelle d'après la formule (BROODY)

$$
\mathrm{S}=0, \mathrm{I} 25 \times \mathrm{P}^{0,56} \text { pour les ovins }
$$

Par ailleurs, nous pouvons connaître, pour chaque race, exprimée en $\%$ de la surface corporelle totale, la fraction de cette surface recouverte par la toison.

Classés par ordre décroissant, nous obtenons les résultats suivants :

Surface $\%$ de la surface totale

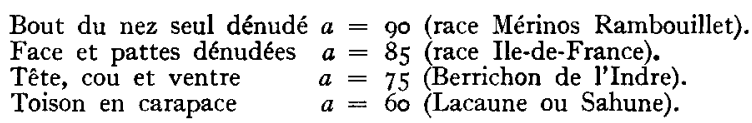

La surface couverte peut s'obtenir en multipliant la surface totale $S$ par le coefficient $a$ de ce tableau.

$$
\mathrm{S}^{\prime}=a \mathrm{~S} .
$$

Du poids de la toison exprimée en laine lavée à fond, nous passons au poids par unité de surface :

$$
\frac{\mathrm{P}}{\mathrm{S}^{\prime}} .
$$

Les mesures effectuées sur des mèches-échantillons prélevées à l'épaule et à la cuisse nous donnent la finesse moyenne $\mu$ et la longueur moyenne $l$ d'une fibre de densité connue $\mathrm{D}=\mathrm{I}, 3 \mathrm{I} \%\left({ }^{\mathbf{1}}\right)$.

La finesse $u$ correspondant au diamètre moyen de la fibre, le poids de celleci s'obtient par la formule

$$
\frac{u^{3}}{4} \times \pi \times l \times \mathbf{I}, 3 \mathbf{I}
$$

On en déduit la valeur $\mathrm{N} d u$ tassé, qui exprime le nombre de fibres par unité de surface, au moyen du calcul ci-après :

$$
\begin{aligned}
\mathrm{N} & =\frac{\frac{\mathrm{P}}{\mathrm{S}}}{\frac{\mu^{2}}{4} \pi l \mathrm{D}} \\
\mathrm{N} & =\frac{4 \mathrm{P}}{a\left(0, \mathrm{I} 25 \times \mathrm{P}^{0,56}\right)} \mu^{2} l \mathrm{D} .
\end{aligned}
$$

Les erreurs les plus importantes sont commises par le calcul de $S$ à partir de $P$ : nous avons personnellement constaté des erreurs atteignant $20 \%$, et dans l'évaluation de $a$.

Le Pr A. M. LERoy a ainsi pu montrer les variations considérables du tassé d'une race à l'autre.

Voici quelques résultats :

(1) Donnée correspondant à la densité de la laine mesurée dans l'air, dans une atmosphère dont le degré d'hygrométrie relative est de $65+2,5 \%$. 


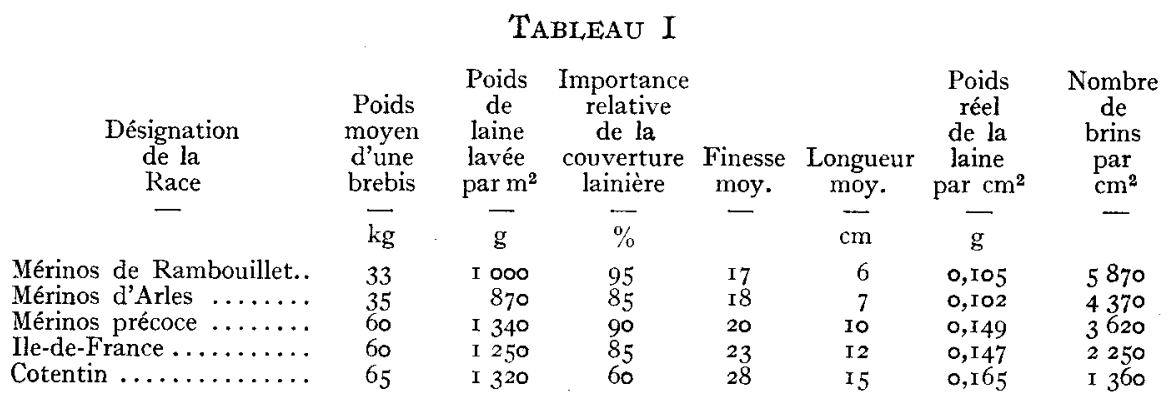

Il existe d'autres méthodes indirectes, mais leurs résultats sont toujours approchés.

\section{MÉTHODÉS DIRECTES}

L'on peut concevoir deux types de méthodes directes : l'un fait appel au tatouage, l'autre à l'emploi d'appareils spécialement conçus.

\section{I. - Méthode par tatouage}

La méthode par tatouage a été mise au point en Angleterre par HaMMARD et GALPIN. L'appareil se compose d'une petite plaque de métal, garnie de pointes acérées d'environ I $\mathrm{cm}$ de long, disposées selon les côtés et les diagonales d'un carré.

4 secteurs égaux $A, B, C, D$ sont ainsi délimités.

On tatoue, après la tonte, cette figure en divers points, judicieusement choisis, de la pean de mouton.

Pour savoir quel est le nombre de fibres sur chacun des emplacements dessinés par ce moyen, il suffit de couper avec soin, aussi près que possible de la peau, tous les brins de laine d'un secteur.

L'existence des autres secteurs permet de faire d'autres prélèvements sur des surfaces identiques, mais à d'autres intervalles de temps.

Par ce procédé, les prélèvements successifs, s'ils s'effectuent toujours sur la même portion de peau, ne correspondent pas toujours à des surfaces rigoureusement égales, principalement s'il s'agit d'un animal en croissance.

Sur les adultes, il est possible d'observer des déformations de la figure, qui rendent inegales les surfaces des divers secteurs. Ajoutons que ce tatotage ne peut pas se faire dans le cas de toisons denses dont les fibres ont déjà quelques centimètres de longueur.

Enfin, les Eleveurs n'aiment pas beaucoup que l'on tatoue leurs animaux, car il peut en résulter une dépréciation de valeur marchande. Or, il est souvent nécessaire de travailler sur des troupeaux privés. On peut disposer, ainsi, d'un cheptel abondant, renouvelé à chaque nouvelle série de recherches, générale- 
ment homogène et de bonne qualité ; il ne serait pas possible d'utiliser un aussi grand nombre de sujets en se bornant à des observations sur un troupeau expétimental.

\section{2. - Utilisation d'appareils spéciaux}

Pour les raisons précédemment exposées, nous avons été conduits, depuis I944, à étudier un appareil permettant de mesurer le tassé sans faire appel au tatouage.

Nous avons d'abord utilisé un premier appareil, qui nous a servi à mettre au point le type définitif. Nous décrirons successivement les deux appareils.

Nous avons pensé qu'une surface carrée, de $\mathrm{I} / 4 \mathrm{de} \mathrm{cm}^{2}$, pourrait convenir pour ce genre d'étude, car elle permet d'opérer des prélèvements nombreux, sans pour cela laisser, sur l'animal en laine, des traces trop apparentes.

\section{Type primitif}

Description : Le premier appareil se composait de 2 lames minces d'acier de $8 /$ Io de $\mathrm{mm}$, hautes de $\mathrm{I}$ à $2 \mathrm{~cm}$, et longues de $3 \mathrm{~cm}$, maintenues parallèles et à un écartement de $\mathrm{I} / 2 \mathrm{~cm}$, par rivetage sur une tige carrée formant manche. A I $/ 2 \mathrm{~cm}$ de la face intérieure de cette tige, une encoche sur chaque lame permettait d'enfoncer une troisième lame, délimitant ainsi le $4^{\mathrm{e}}$ côté du I/4 de $\mathrm{cm}^{2}$ (fig. I).

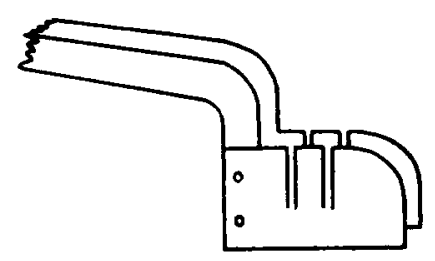

Fig. I.

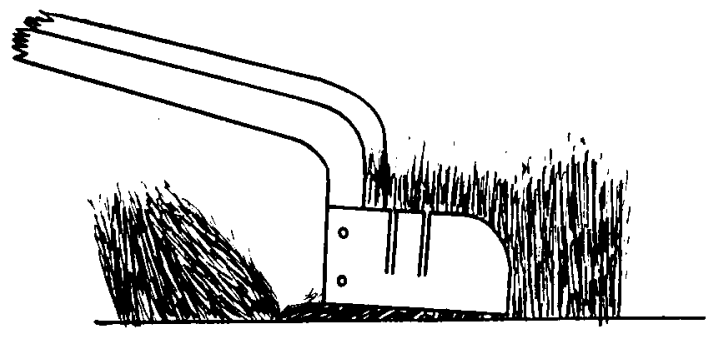

Fig. 2.

Mode opératoire : $\mathbf{I}^{\mathrm{o}}$ Ouvrir la toison avec les mains, en lissant les bords de la fente avec la main posée à plat.

$2^{\circ}$ Enfoncer à fond l'appareil comme un peigne dans l'une de ces lèvres ou bordure de laine, en frottant le bord des lames contre la peau. Il faut que ces lames soient aussi perpendiculaires que possible au bord des lèvres (fig. 2).

$3^{\circ}$ Séparer une mèche de $\mathrm{r} / 2 \mathrm{~cm}$ de large et de la longueur des dents par un léger mouvement vertical de peignage.

$4^{\circ}$ Délimiter, dans cette mèche, une longueur de $\mathrm{r} / 2 \mathrm{~cm}$, en enfonçant la $3^{\mathrm{e}}$ lame dans les encoches. On sépare, par un léger mouvement de peignage cette mèche, que l'on coupe aussi près que possible de la peau. 


\section{Inconvénients}

Après usage, nous nous sommes aperçus que l'on pouvait faire, à l'emploi de cet instrument, les critiques suivantes :

I ${ }^{\circ}$ Erreur de largeur. - L'écartement des deux lames minces ne peut être maintenu rigoureusement constant, malgré l'insertion entre elles d'un morceau de bois de dimensions convenables pour les empêcher de s'écarter pendant le transport. Les erreurs entraînées par cette inégalité d'écartement peuvent atteindre $\mathrm{I} / 2 \mathrm{~mm}$ en largeur pour une mèche.

$2^{\circ}$ Rupture de fibres. - Le peignage des mèches longues et tassées par des lames étroites rompt aisément des fibres un peu entremêlées à leur partie terminale. De plus, les encoches à bords vifs peuvent arracher des fibres qui échappent ensuite à la mesure.

$3^{\circ}$ Erreur de perpendicularité. - Au cours du deuxième temps, un manque de perpendicularité entre la direction des lames et le bord de la fente dans la toison peut aussi entraîner une erreur (fig. 3). Dans ce cas, les prélèvements sont faits sur une surface trapézoïdale, et non pas sur la surface carrée désirée.
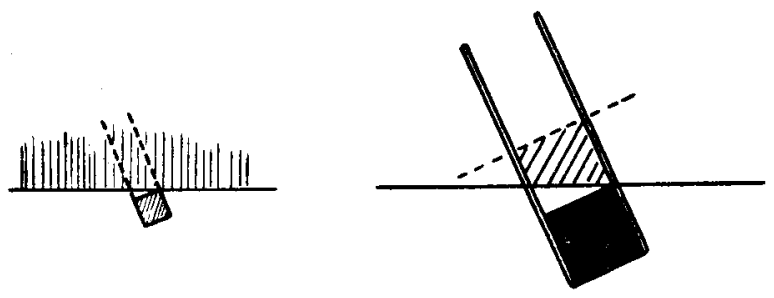

Fig. 3.

$4^{0}$ Erreur de longueur. - La mise en place de la lame séparatrice peut provoquer un mouvement de l'appareil, d'où un excès de longueur de $\mathrm{I} / 2 \mathrm{~mm}$.

Nous avons été obligés de renoncer à ce type d'appareil dont la précision, suffisante dans certains cas, ne convenait pas aux travaux que nous désirons poursuivre par la suite.

\section{Type définitif}

Nous avons été amenés aux modifications suivantes :

Renforcement des dents formées de deux tiges dont la section a la forme d'r/4 de cercle arrondie en dessus, ce qui supprime les encoches. La rigidité de l'appareil est remarquable. Après 2 ans d'usage, nous n'avons pas constaté de variation dans l'écartement des dents. Leur hauteur moindre $-1 / 2 \mathrm{~cm}$ 
à peine - permet de travailler avec des toisons ayant moins de $2 \mathrm{~cm}$ de longueur (fig. 4).

Le risque de rupture des fibres, signalé au paragraphe 3, est nettement diminué par le nouveau profil et la suppression des encoches.

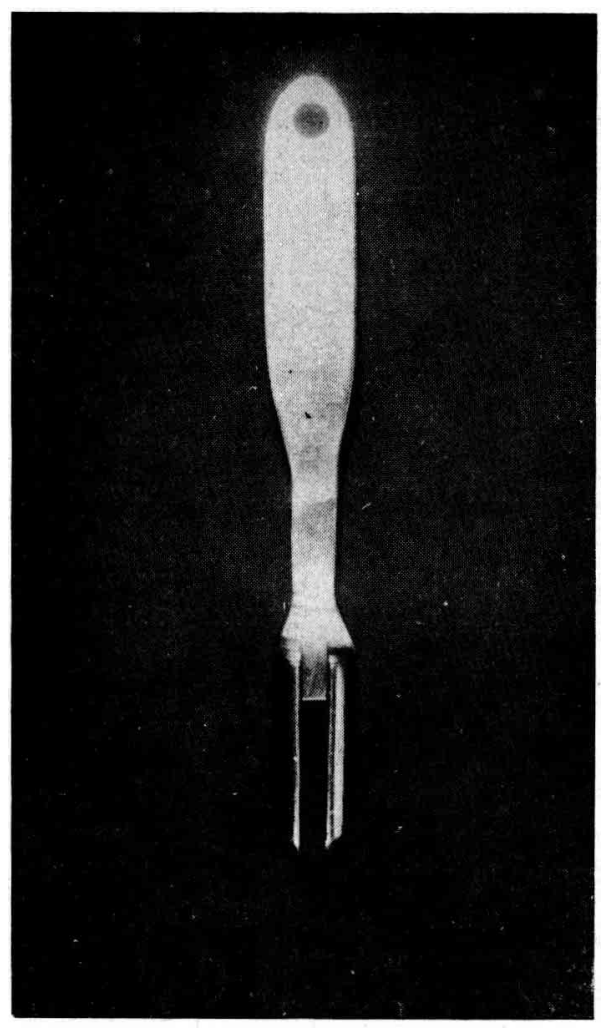

Fig. 4 .

Cette suppression des encoches et la nécessité d'éviter l'erreur due à un enfoncement irrégulier, nous a conduits à modifier la méthode (fig. 5).

Dans le temps $\mathrm{n}^{\mathrm{o}} 2$, on enfonce l'appareil sans avoir à se préoccuper de la direction ou du degré d'enfoncement.

Le temps $n^{0} 3$ ne subit pas de modification. Il consiste à délimiter une mèche allongée de $\mathrm{I} / 2 \mathrm{~cm}$ de large et à dégager cette mèche des fibres avoisinantes.

En maintenant par son extrémité la mèche ainsi délimitée entre le pouce et l'index de la main gauche, on retire l'appareil puis on l'enfonce perpendiculairement à la première direction. Ainsi se trouve délimitée avec précision une mèche de $\mathrm{I} / 2 \mathrm{~cm}$ de côté.

La seule cause d'erreur réside ici dans le fait que l'appréciation d'une 
direction perpendiculaire à la première se fait au jugé. Mais cette cause d'erreur est pratiquement insignifiante.

Toutes les autres causes d'erreur précédemment décrites ont été supprimées.

Depuis 4 ans que nous 1'utilisons, l'appareil s'est montré solide, précis et d'une grande simplicité d'emploi.

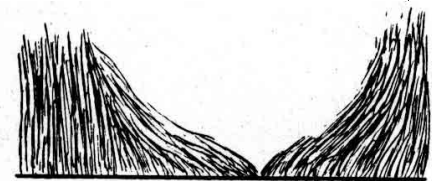

1

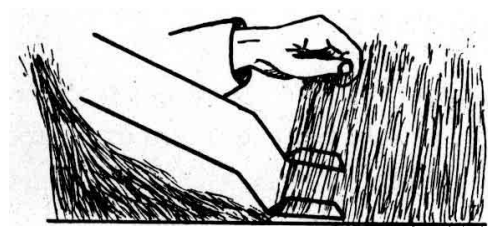

3

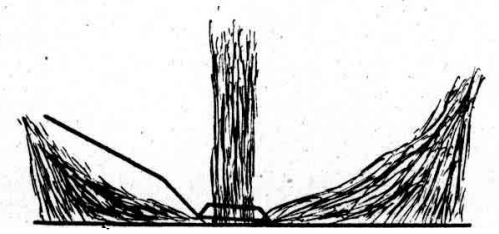

5
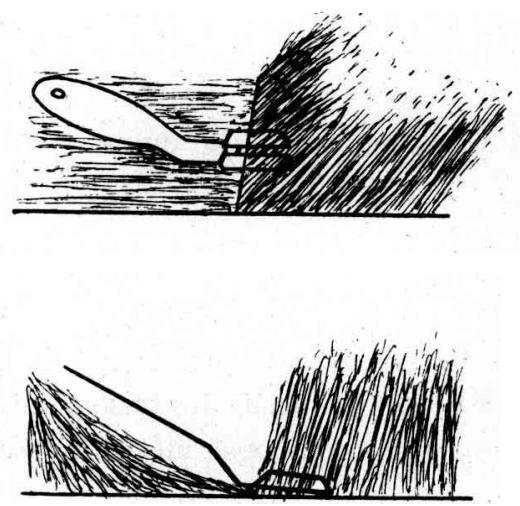

2 et 2 bis

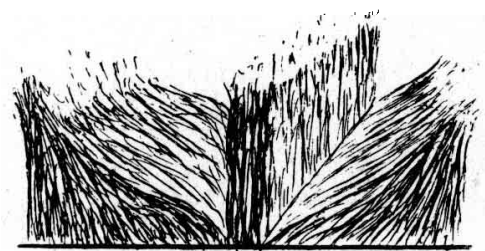

4

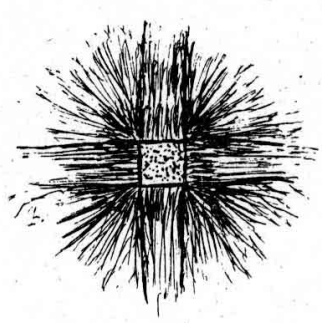

6

Fig. 5.

Numération : La méthode de numération est la même avec les deux appareils. On pèse la mèche obtenue, soit $\mathrm{P}$, on en sépare une partie de poids $p$. On compte les fibres de cette partie choisie aussi petite que possible pour simplifier les numérations. Par exemple, s'il s'agit d'une toison fine, de type Mérinos, l'échantillon destiné à être compté correspond au I/4 de l'échantillon total. Lorsqu'il s'agit d'une toison relativement grossière, comme celle des 
moutons Texel, l'échantillon sera plus important. Soit $n$, le nombre de fibres ainsi trouvé. Le nombre de fibres par $\mathrm{cm}^{2}$ sera :

$$
\mathrm{N}=n \times \frac{\mathrm{P}}{\mathrm{p}} \times 4 .
$$

Poids de toison par unité de surface : Le poids de toison en $\mathrm{kg}$ par $\mathrm{m}^{2}$ sera égal à :

$$
\frac{\mathrm{P} \times 4 \times \mathrm{IO}^{4}}{3}=40 \times \mathrm{P}
$$

$P$ étant exprimé en grammes.

\section{QUELQUES RESULTATS}

Il nous a paru intéressant de donner quelques résultats obtenus par cette méthode directe sur des toisons de différentes races. On peut les comparer aux résultats obtenus par méthode indirecte (tableau II).

\begin{tabular}{|c|c|c|}
\hline Désignation de la race & $\begin{array}{l}\text { Poids réel de laine } \\
\text { par } \mathrm{cm}^{2}\end{array}$ & $\begin{array}{l}\text { Nombre de brins de laine } \\
\text { par } \mathrm{cm}^{2}\end{array}$ \\
\hline 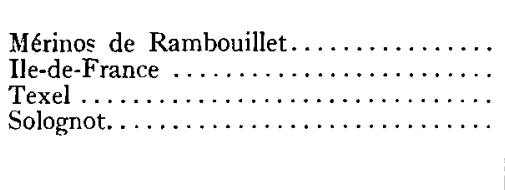 & $\begin{array}{c}\mathrm{g} \\
0, \mathrm{I} 55 \mathrm{I} \\
0, \mathrm{I} 202 \\
0, \mathrm{I} 604 \\
\text { o,078r } \\
\text { o, y compris jusqu'à } 25 \% \\
\text { de jarres) }\end{array}$ & $\begin{array}{r}4 \cdot 794 \\
2.678 \\
\mathrm{I} \cdot 53^{8} \\
924\end{array}$ \\
\hline
\end{tabular}

TABLEAU II

\section{Conclusions}

D'après ce qui précède, nous sommes amenés à penser que la mesure du tassé, par la méthode directe, doit être préférée à la méthode indirecte, tant en raison de sa commodité d'emploi que de sa plus grande précision.

\section{INFL UENCE DU POIDS DE LAINE PAR UNITÉ DE SURFACE SUR LE POIDS TOTAL DES TOISONS}

Le rôle du tassé et du poids de laine par unité de surface dans le poids des toisons a été souvent cité, par DUERDEN notamment. Il nous a paru intéressant d'appliquer, dès sa mise au point, la méthode précédente à cette étude. 
Nous avons recherché une race et, au sein de celle-ci, un troupeau très homogène quant à la surface couverte par la toison. Dans ce troupeau inscrit de la race Texel vivant en plein air, mode de vie qui élimine une partie des variations de rendement en augmentant fortement celui-ci, nous avons choisi un certain nombre d'animaux d'âge et de sexe différents: brebis, agnelles, béliers. Le but a été de voir s'il existait une corrélation entre le poids de laine par unité de surface et le poids de la toison à la tonte.

Méthodes : Juste avant la tonte, les prélèvements d'échantillons ont été effectués à l'aide de l'appareil précédemment décrit. L'endroit choisi était le flanc de l'animal, au niveau de la moitié de la dernière côte, région où les caractéristiques des fibres se rapprochent le mieux de celles de l'ensemble de la toison $\left(^{1}\right)$. Celle-ci était pesée aussitôt après la tonte. Les échantillons étaient pesés le lendemain, dès leur arrivée au Laboratoire.

Résultats : Le tableau suivant expose les résultats obtenus.

TABLEAU III

\begin{tabular}{|c|c|c|c|c|c|}
\hline $\begin{array}{l}\text { No des } \\
\text { Animaux }\end{array}$ & $\begin{array}{l}\text { Poids du } \mathrm{I} / 4 \text { de } \mathrm{cm}^{2} \\
\text { au flanc de l'animal }\end{array}$ & $\begin{array}{l}\text { Poids de } \\
\text { Toison }\end{array}$ & $\begin{array}{l}\text { No des } \\
\text { Animaux }\end{array}$ & $\begin{array}{l}\text { Poids du } \mathrm{I} / 4 \mathrm{de} \mathrm{cm}^{2} \\
\text { au flanc de l'animal }\end{array}$ & $\begin{array}{c}\text { Poids de } \\
\text { Toison }\end{array}$ \\
\hline & $\mathrm{g}$ & $\mathrm{kg}$ & & $g$ & $\mathrm{~kg}$ \\
\hline I $\ldots \ldots$. & 0,1663 & 4,700 & I 6 & 0,0390 & 2,500 \\
\hline $2 \ldots \ldots \ldots$ & 0,0950 & 5,000 & I 7 & 0,0474 & 3,400 \\
\hline $3 \ldots \ldots$ & $0,142 \mathrm{I}$ & 6,500 & I8 & 0,1194 & 4,100 \\
\hline $4 \ldots \ldots \ldots$ & 0,1624 & 6,250 & 19 & 0, I I I 2 & $4,35^{\circ}$ \\
\hline $7 \ldots \ldots$ & 0,0900 & $3,55^{\circ}$ & 20 & 0,1903 & 6,250 \\
\hline $8 \ldots \ldots \ldots$ & $0,105 \mathrm{r}$ & 3,650 & $2 \mathrm{I}$ & 0,1007 & 4,000 \\
\hline $9 \ldots \ldots$ & 0,0792 & 2,700 & 22 & 0,1418 & 5,100 \\
\hline ro....... & 0,0958 & 4,750 & 23 & $0,135^{\mathrm{I}}$ & 5,800 \\
\hline II $\ldots \ldots \ldots$ & 0,1 I I 7 & 3,850 & 24 & 0,1039 & 5,200 \\
\hline $12 \ldots \ldots \ldots$ & 0,1247 & 3,250 & 25 & $0,104 \mathrm{I}$ & 4,600 \\
\hline $13 \ldots \ldots$ & 0,1 I 24 & 3,750 & 26 & 0,1759 & 7,000 \\
\hline $14 \ldots \ldots$ & $0,06 \mathrm{I} 8$ & 3,275 & 27 & 0, I I 50 & 4,300 \\
\hline $15 \ldots \ldots$ & 0,0890 & 3,350 & & & \\
\hline
\end{tabular}

Le coefficient de corrélation entre le poids de laine par unité de surface et le poids total des toisons atteint $0,74 \pm 0,09$ résultat hautement significatif. L'écart indique une bonne homogénéité, si l'on tient compte du fait que les toisons ont été pesées à l'état brut, avec leur suint et les impuretés qu'elles retiennent.

Conclusions: Dans le cas d'un ensemble d'animaux de race pure, c'està-dire ayant des caractéristiques lainières homogènes et vivant dans des conditions identiques afin d'éliminer les différences dues aux modes de vie, il semble que c'est la quantité de laine par unité de surface qui conditionne principalement le poids de la toison.

Des travaux précédents ont mis en évidence que le caractère " poids

(1) Etudes sur la topographie de la toison. Travaux non publiés. 
de toison par unité de surface " peut se transmettre par le mécanisme habituel de 1'hérédité.

Jusqu'ici, pour apprécier ce caractère, il fallait peser la toison puis 1'animal ; ce dernier poids donnait la surface corporelle approchée, d'où l'on déduisait la surface couverte. Ces pesées ne sont pas toujours faciles et parfois impossibles à mener à bien pour un nombre suffisamment élevé d'animaux dans un troupeau privé ; d'autre part, elle comporte une marge d'erreur considérable atteignant parfois $20 \%$.

Au contraire, 1'utilisation de notre appareil permet de contrôler un grand nombre d'animaux sans manipulations difficiles et donne des résultats nettement plus précis.

Ainsi, si les premières données sont confirmées par la suite pour d'autres races, elles pourront permettre d'effectuer sur des bases précises des calculs d'héritabilité et ainsi, concourir à orienter la sélection.

\section{BIBLIOGRAPHIE}

Charlet (P.). - Études sur la topographie de la toison. Travaux non publiés.

DavenPort (C. B.) et Ritzman (E. G.). - Some wool characters and their inheritance. New Hants Agric. Sta. Bull., 31, p. 58, 1926.

DVERDEN (J. E.). - Relation of density of wool to surface area in the growing sheep, $S$. Africa J. Sci., I925; Absence of uniformity in growth of the merinos fleece. $J$. Text. Inst., 18, p. I9I-I94, 1927; Evolution of the fleece of the sheep. J. Afric. J. Sci., 24, p. $388-415$, I927.

HILL (J. A.). - Studies in the variation and correlation of fleece from Range sheep. Univ. Wyo. Agri. Exp. Sta. Bull., 12\%, p. 39-52, г92 г.

Leroy (A.-M.). - Le Mouton. Éd. Hachette, Paris, p. 228.

LUSH (J. L.) et JONES (J. N.). - The influence of individuality age and season upon the weight of fleeces produced by Range sheep. Texas Agric. Exp. Sta. Bull., 311, p. 45, I923.

Calpin (N.) et Hammond. - Cité par Hammond: Sheep Breeding in Great Britain. 